\title{
Multiple-Input Multiple-Output Generalized Frequency Division Multiplexing with Index Modulation
}

\author{
Ersin Öztürk ${ }^{\mathrm{a}, \mathrm{b}, *}$, Ertugrul Basar ${ }^{\mathrm{c}}$, Hakan Ali Çırpan ${ }^{\mathrm{a}}$ \\ ${ }^{a}$ Istanbul Technical University, Faculty of Electrical and Electronics Engineering, 34469, \\ Maslak, Istanbul, Turkey \\ ${ }^{b}$ Netas, Department of Research and Development, 34912, Pendik, Istanbul, Turkey \\ ${ }^{c}$ Communications Research and Innovation Laboratory (CoreLAB), Department of \\ Electrical and Electronics Engineering, Koç University, Sariyer 34450, Istanbul, Turkey
}

\begin{abstract}
The demand for wireless access continues to grow with the new applications which create a broad range of technical challenges. Although orthogonal frequency division multiplexing (OFDM) with multiple numerologies concept will likely address the current technical challenges of fifth generation (5G) wireless networks, the sufficiency of OFDM-based physical layer (PHY) is quite disputable due to massive growth trend on the number of wireless users and applications for future wireless networks. Therefore, enhanced radio access technologies (RATs) are needed to fulfill the technical requirements of beyond $5 \mathrm{G}$ networks. Generalized frequency division multiplexing (GFDM) has attracted tremendous attention over the past few years because of its advantages in terms of out-of-band (OOB) emission, spectral efficiency and latency. Index modulation (IM) techniques convey digital information by utilizing transmission entities in an innovative way and offer attractive advantages such as energy and spectral efficiency without increasing the computational complexity. On the other hand, multiple-input multiple-output (MIMO) transmission is an unquestionable technology to enable increased spectral efficiency. In this paper, a novel MIMO-GFDM scheme, which combines spatial multiplexing (SMX) MIMO transmission, GFDM and IM, is proposed in order to provide an effi-
\end{abstract}

\footnotetext{
* Corresponding author
} 
cient transmission scheme for beyond 5G wireless networks. A minimum mean squared error (MMSE)-QR decomposition-based near-optimum detector is proposed for the receiver side and bit error rate, OOB emission, spectral efficiency and computational complexity of the proposed scheme are compared with classical SMX-OFDM and SMX-GFDM schemes via computer simulations. It has been demonstrated that the proposed SMX-GFDM-IM scheme can be considered as a viable PHY scheme for beyond 5G wireless networks.

Keywords: GFDM, MIMO systems, spatial multiplexing, index modulation, $5 \mathrm{G}$ wireless networks, multicarrier modulation, physical layer design.

\section{Introduction}

As the number of mobile devices and users has increased tremendously, the demand for wireless access continues to grow with the new applications spanning from machine type communications to wireless regional area networks. Since these applications create a broad range of technical challenges, such as high spectral and energy efficiency, less out-of-band emission (OOB), low latency and low peak-to-average power ratio (PAPR), which go beyond fourth generation (4G) wireless networks [1, standardization activities for the cellular infrastructure are moving towards fifth generation $(5 \mathrm{G})$ wireless networks [2].

Orthogonal frequency division multiplexing (OFDM), which forms the physical layer (PHY) of $4 \mathrm{G}$ wireless networks, has attractive advantages such as simple implementation via fast Fourier transform (FFT)/inverse FFT (IFFT) blocks, robustness to frequency selective fading and simple equalization. However, considering the diverse applications of future wireless networks, the shortcomings of OFDM such as high PAPR, high OOB emission and sensitivity to time and frequency synchronization errors make it difficult to address the requirements of the future radio access technologies $[3,4]$. Therefore, radically new PHY technologies and modulation formats have been explored to fulfill the challenges of $5 \mathrm{G}$ wireless networks [3]. After years of discussion on the standardization of $5 \mathrm{G}$, it was decided that none of the waveform alternatives were 
able to address all the requirements at the same time and an expanded version of OFDM with multiple numerologies, i.e., different parameterization of OFDM subframes, would provide the optimum results for the PHY of 5G [5, 6].

Considering the massive growth trend from first generation $(1 \mathrm{G})$ to $5 \mathrm{G}$, it 25 is expected that the number of wireless users and applications will certainly continue to increase beyond $5 \mathrm{G}$ with expanded diverse requirements and technical challenges. For this reason, although OFDM with multiple numerologies concept will likely address the current technical challenges of $5 \mathrm{G}$ wireless networks, the sufficiency of OFDM-based PHY is quite disputable for beyond 5G. Consequently, enhanced radio access technologies (RATs) are needed to fulfill the technical challenges of beyond $5 \mathrm{G}$ wireless networks.

Generalized frequency division multiplexing (GFDM) [7], which has attracted tremendous attention over the past few years, is a PHY scheme proposed for future wireless networks. It comes into prominence by providing advantages in terms of OOB emission, spectral efficiency and latency due to digitally pulse shaping of each subcarrier, reduced overhead of cyclic prefix and block-based structure, respectively. Unlike OFDM, GFDM permits to use more than one timeslot on the subcarriers and enables flexible time-frequency structuring which can be shaped according to corresponding scenario. Therefore, GFDM is one of 40 the candidate schemes for beyond $5 \mathrm{G}$ wireless networks.

Multiple-input multiple-output (MIMO) transmission is an unquestionable method to enable increased spectral efficiency. For this reason, applicability with MIMO techniques is mandatory for a candidate PHY scheme for future wireless networks. In this sense, the combination of GFDM with spacetime coding (STC) technique [7, 8, 9, 10, and spatial multiplexing (SMX) [1, 12, 13, 14, 15, 16, 17, 18, 19, have been investigated. In [11, 12, 13, iterative algorithms have been proposed for detecting spatially multiplexed GFDM signals. In [14, linear MIMO detection algorithms such as minimum mean squared error (MMSE) and zero-forcing (ZF) have been investigated. In [15], GFDM modulation and MIMO transmission have been combined as an equivalent MIMO-GFDM channel and QR decomposition-based sphere decoding (SD) 
with successive interference cancellation (SIC) has been proposed. Coded performance analysis of QR decomposition-based SD-SIC receiver has been presented in [16]. In [17] and [18, an iterative MMSE with parallel interference cancellation (MMSE-PIC) detection has been proposed for MIMO-GFDM. Furthermore, a low complexity implementation of MMSE equalization has been proposed in [19]. Among these studies, it has been reported that GFDM performed worse than OFDM in [14] and [16]. Based on these obtained results, while non-iterative MIMO-GFDM receivers generally performed worse than OFDM, iterative MIMO-GFDM receivers outperformed OFDM schemes at the cost of extra processing latency. Nevertheless, despite these advancements, nonorthogonality resulting from filtering each subcarrier individually prevents the frequency domain decoupling and makes the MIMO detection complicated.

Index modulation (IM) techniques convey digital information by utilizing transmission entities in an innovative way and offer attractive advantages such as energy and spectral efficiency without increasing the computational complexity [20. IM schemes transmit information through alternative ways. While the on/off status of the transmit antennas are altered according to information bits in spatial modulation (SM) 21, the on/off status of the subcarriers are altered in OFDM with subcarrier IM 22, 23, 24]. Therefore IM schemes save the transmission energy by deactivating some transmit entities and have the ability to improve error performance by using the saved transmission energy. Since deactivating some transmit entities causes throughput loss, a dual-mode OFDM-IM scheme [25], which uses two different constellation modes, and multiple-mode 75 IM scheme 26, which uses multiple distinguishable constellations, have been proposed to prevent throughput loss still using transmit indices to convey extra information. Recently, several studies have been proposed to improve the coding gain, diversity gain and spectral efficiency of OFDM-IM [27, 28, 29, 30, 31]. Furthermore, applicability of MIMO methods along with the OFDM-IM technique so has been analyzed in $32,33,34,35$, and significant performance gains have been reported. Taking account the prominent advantages of IM [36], combination of GFDM with IM techniques has been investigated in [37, 38, 39, 40, 41, 42, 43. 
In [37, the combination of GFDM with SM has been considered by using a ZF-based suboptimal receiver. In [38, GFDM has been combined with subcarrier IM technique and the combined application of space and frequency IM with GFDM has been investigated in 39]. In [40], a framework for the combination of GFDM with IM has been presented and several GFDM-IM schemes have been compared in terms of computational complexity, spectral efficiency and bit error rate (BER). In [41, flexible IM numerology has been proposed to reduce OOB emission of GFDM further and a mechanism to optimize OOB emission, spectral efficiency and latency has been provided. In [42], the combination of Kalman algorithm-based channel estimation, turbo MMSE equalization and phase rotated conjugate cancellation (PRCC) algorithm has been proposed for MIMO-GFDM-IM scheme. In [43, a GFDM-IM transceiver structure has been introduced for multi-user networks and BER performance improvement with respect to classical GFDM and OFDM-IM has been reported.

Against this background, in this paper, a novel MIMO-GFDM system, which combines SMX, GFDM and IM, is proposed in order to provide an efficient transmission scheme for beyond $5 \mathrm{G}$ wireless networks. The proposed scheme transmits independent GFDM-IM blocks through each transmit antenna and adapts minimum mean squared error (MMSE)-QR decomposition-based nearoptimum detector at the receiver side. To the best of authors' knowledge, this application would be the first attempt to exploit a near-optimum detector with SMX-GFDM-IM system. We analyze the uncoded and coded BER, OOB emission, spectral efficiency and computational complexity of the proposed scheme by comparing with classical SMX-OFDM and SMX-GFDM schemes via computer simulations. It has been demonstrated that the proposed SMX-GFDM-IM scheme can be considered as a viable PHY scheme for beyond 5G wireless networks due to its improved BER, OOB emission and spectral efficiency.

110 The remaining sections are organized as follows. The system models of the SMX-GFDM-IM system is presented in Section II. In Section III, computational complexity of the proposed SMX-GFDM-IM scheme is analyzed. Simulation results concerning the BER performance, computational complexity, spectral 
efficiency and OOB emission of the proposed SMX-GFDM-IM scheme are presented in Section IV. Finally, Section V concludes the paper ${ }^{1}$

\section{System Model of SMX-GFDM-IM}

\subsection{SMX-GFDM-IM Transmitter}

In this study, a GFDM-based SMX system with $T$ transmit and $R$ receive antennas is considered. The block diagram of the SMX-GFDM-IM transmit-

\footnotetext{
${ }^{1}$ Notation: Column vectors and matrices are denoted by boldface lowercase and capital letters, respectively. $(\cdot)^{T}$ and $(\cdot)^{H}$ denote transposition and Hermitian transposition of a vector or a matrix, respectively, and $(\cdot)^{-1}$ indicates the inverse of a matrix. $C(\alpha, \beta)$ denotes the binomial coefficient and $\lfloor\cdot\rfloor$ is the floor function. $X \sim \mathcal{C N}\left(0, \sigma_{X}^{2}\right)$ represents the distribution of a circularly symmetric complex Gaussian random variable $X$ with variance $\sigma_{X}^{2} \cdot\|\cdot\|$ stands for the Euclidean norm. $\mathcal{S}$ denotes $Q$-ary signal constellation and $\mathbf{x}(a: b)$ stands for all elements of $\mathbf{x}$ with indices from $a$ to $b$, inclusive of $a$ and $b$.
} 


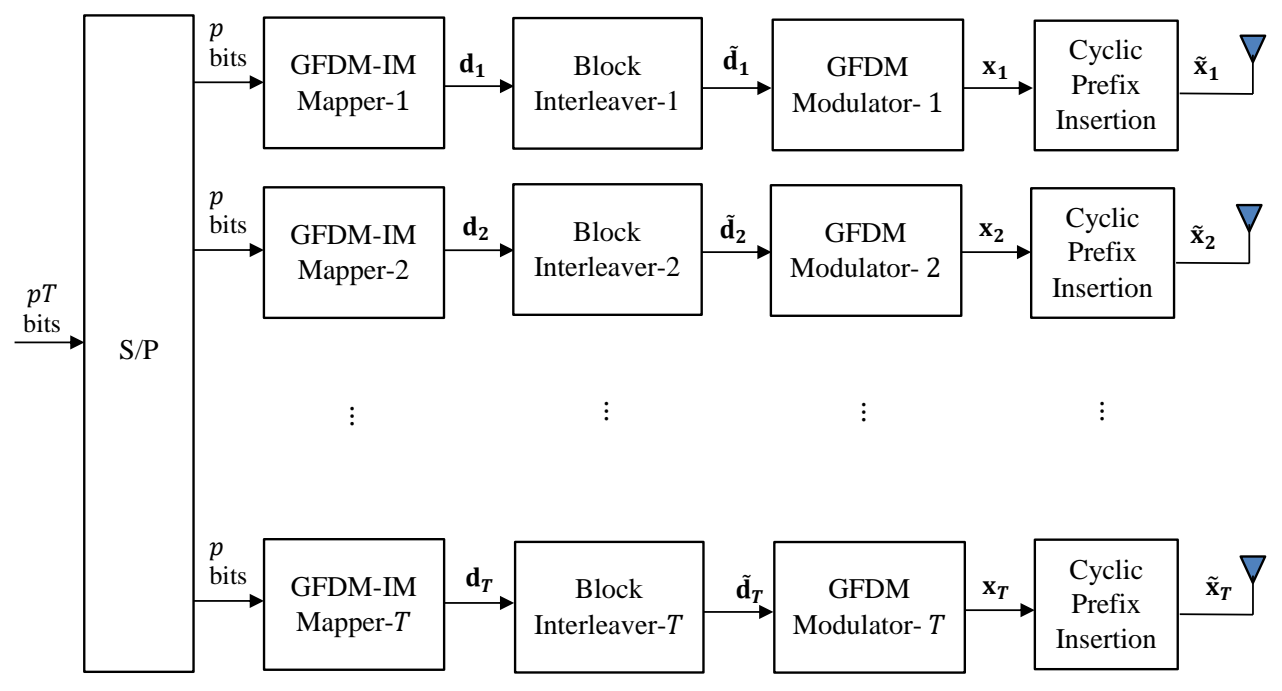

Figure 1: Block diagram of the SMX-GFDM-IM transmitter.

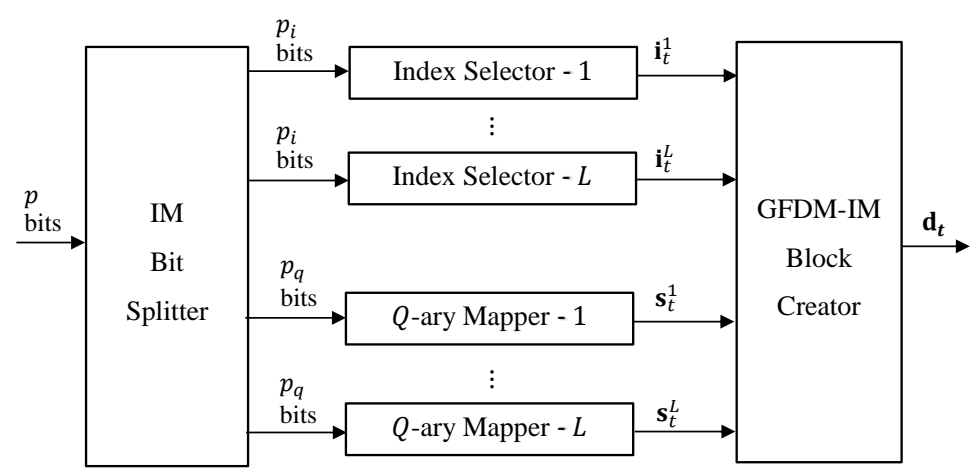

Figure 2: GFDM-IM mappers at each branch of the transmitter.

each GFDM-IM subblock $l$ of $t$ th transmit antenna, only $v$ out of $u$ available subcarriers are chosen as active, where the chosen subcarrier indices are denoted by $\mathbf{i}_{t}^{l}=\left[i_{t}^{l}(1), i_{t}^{l}(2), \ldots, i_{t}^{l}(v)\right]^{T}, i_{t}^{l}(\gamma) \in\{1,2, \ldots, u\}$, for $\gamma=1,2, \ldots, v$, and the remaining $u-v$ subcarriers are set to zero. Therefore, $\mathbf{i}_{t}^{l}$ has $c=2^{p_{i}}$ possible realizations and $p_{i}$ can be defined as $\left\lfloor\log _{2}(C(u, v))\right\rfloor$. Meanwhile, $p_{q^{-}}$ bit sequences are mapped by $Q$-ary mappers to create the modulated symbols vector $\mathbf{s}_{t}^{l}=\left[s_{t}^{l}(1), s_{t}^{l}(2), \ldots, s_{t}^{l}(v)\right]^{T}$, where $s_{t}^{l}(\gamma) \in \mathcal{S}$, for $\gamma=1,2, \ldots, v, l=$ 
$1,2, \ldots, L$. For each GFDM-IM subblock $\mathbf{d}_{t}^{l}, p_{q}=v \log _{2}(Q)$ bits are conveyed by $v$ elements of $s_{t}^{l}$ whose corresponding subcarrier indices given by $\mathbf{i}_{t}^{l}$. After that, in each branch of the transmitter, GFDM-IM block creator constructs the GFDM-IM subblocks $\mathbf{d}_{t}^{l}$ by using $\mathbf{s}_{t}^{l}$ and $\mathbf{i}_{t}^{l}$ for all $l$ first and then concatenates these $L$ IM subblocks to obtain the GFDM-IM block $\mathbf{d}_{t}$. At this point, a block interleaver with size $L \times u$ is employed for $\mathbf{d}_{t}$ to obtain uncorrelated channels and the interleaved data vector $\tilde{\mathbf{d}}_{t}$ is obtained. Then, $\tilde{\mathbf{d}}_{t}$ is modulated using a GFDM modulator and the resulting GFDM transmit signal of $t$ th transmit antenna is given by

$$
x_{t}(n)=\sum_{k=0}^{K-1} \sum_{m=0}^{M-1} \widetilde{d}_{t, k, m} g_{k, m}(n),
$$

where $n \in\{0, \ldots, N-1\}$ is the sampling index and

$$
g_{k, m}(n)=g\left((n-m K)_{\bmod N}\right) \exp \left(j 2 \pi \frac{k n}{K}\right)
$$

is the pulse shaping filter circularly shifted to the $m$ th timeslot and modulated to the $k$ th subcarrier. (1) can be reformulated as $\mathbf{x}_{t}=\mathbf{A} \tilde{\mathbf{d}}_{t}$ [7. Here, $\mathbf{A}$ is an ${ }_{155} N \times N$ GFDM modulation matrix given by

$$
\mathbf{A}=\left[\mathbf{g}_{0,0}, \ldots, \mathbf{g}_{K-1,0}, \mathbf{g}_{0,1}, \ldots, \mathbf{g}_{K-1,1}, \ldots, \mathbf{g}_{K-1, M-1}\right]
$$

where $\mathbf{g}_{k, m}=\left[g_{k, m}(0), \ldots, g_{k, m}(N-1)\right]^{T}$ is a vector constructed with pulse shaping filter samples. Finally, in order to make the convolution with the channel circular, a cyclic prefix $(\mathrm{CP})$ with length $N_{\mathrm{CP}}$ is added to $\mathbf{x}_{t}$ and the resulting vector $\tilde{\mathbf{x}}_{t}=\left[\mathbf{x}_{t}\left(N-N_{\mathrm{CP}}+1: N\right)^{T}, \mathbf{x}_{t}^{T}\right]^{T}$ is sent over a frequency-selective Rayleigh fading channel.

\section{2. $S M X-G F D M-I M$ Receiver}

The block diagram of the SMX-GFDM-IM receiver is given in Fig. 3. After the removal of $\mathrm{CP}$, based on the assumption that perfect synchronization is ensured, CP is longer than the channel delay spread $\left(N_{\mathrm{Ch}}\right)$ and the wireless channel remains constant within one GFDM-IM block, the overall received signal is given by 


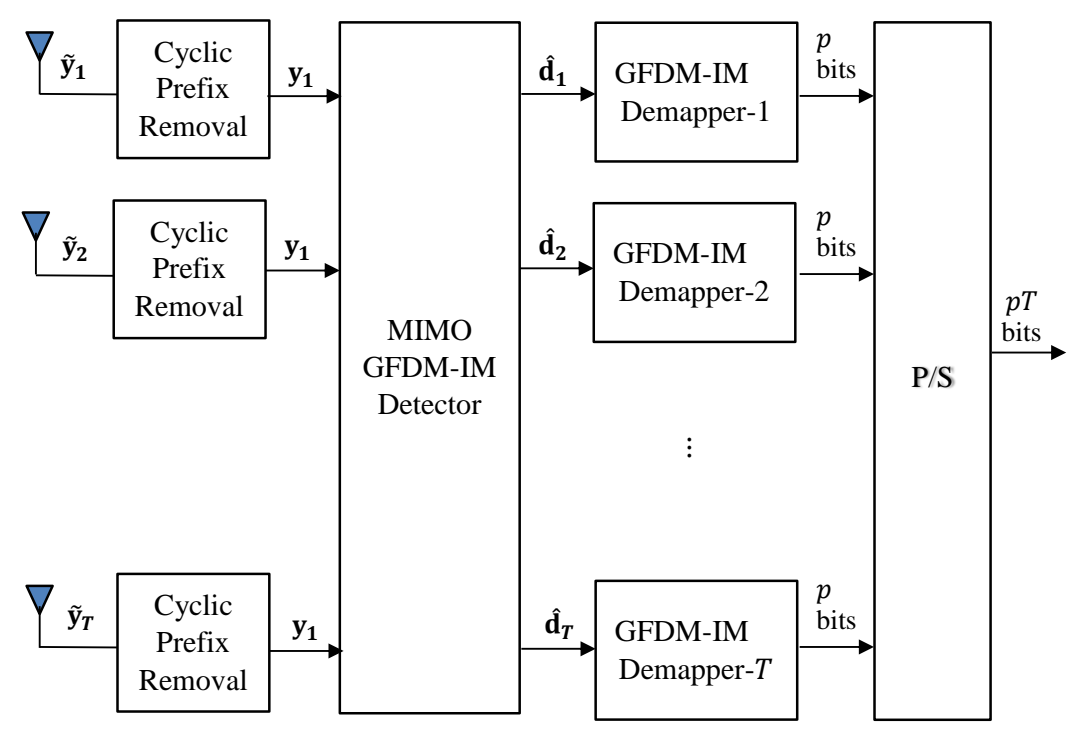

Figure 3: Block diagram of the SMX-GFDM-IM receiver.

$$
\left[\begin{array}{c}
\mathbf{y}_{1} \\
\vdots \\
\mathbf{y}_{R}
\end{array}\right]=\left[\begin{array}{ccc}
\mathbf{H}_{1,1} \mathbf{A} & \cdots & \mathbf{H}_{1, T} \mathbf{A} \\
\vdots & \ddots & \vdots \\
\mathbf{H}_{R, 1} \mathbf{A} & \cdots & \mathbf{H}_{R, T} \mathbf{A}
\end{array}\right]\left[\begin{array}{c}
\widetilde{\mathbf{d}}_{1} \\
\vdots \\
\widetilde{\mathbf{d}}_{T}
\end{array}\right]+\left[\begin{array}{c}
\mathbf{w}_{1} \\
\vdots \\
\mathbf{w}_{R}
\end{array}\right]
$$

where $\mathbf{y}_{r}=\left[y_{r}(0), y_{r}(1), \ldots, y_{r}(N-1)\right]^{T}$ is the vector of the received signals at the $r$ th receive antenna, $\mathbf{H}_{r, t}$, for $r=1, \ldots, R, t=1, \ldots, T$, is the $N \times N$ circular convolution matrix constructed from the channel impulse response coefficients between the th transmit antenna and the $r$ th receive antenna given by $\mathbf{h}_{r, t}=$ $\left[h_{r, t}(0), h_{r, t}(1), \ldots, h_{r, t}\left(N_{\mathrm{Ch}}-1\right)\right]^{\mathrm{T}}$, where $h_{r, t}(n)$ follows $\mathcal{C N}(0,1)$ distribution, $\mathbf{w}_{r}$ is an $N \times 1$ vector of additive white Gaussian noise (AWGN) samples with elements distributed as $\mathcal{C N}\left(0, \sigma_{w}^{2}\right)$. (4) can be rewritten in a more compact form as

$$
\mathbf{y}=\widetilde{\mathbf{H}} \widetilde{\mathbf{d}}+\mathbf{w},
$$

175 where the dimensions of $\mathbf{y}, \widetilde{\mathbf{H}}, \widetilde{\mathbf{d}}$ and $\mathbf{w}$ are $N R \times 1, N R \times N T, N T \times 1$ and $N R \times 1$, respectively. 


\subsubsection{Maximum Likelihood (ML) Detection of SMX-GFDM-IM}

In GFDM, each subcarrier is filtered individually using a prototype filter. While this filtering operation reduces OOB emission, it causes non-orthogonality and intercarrier interference. Therefore, frequency domain decoupling of GFDM subcarriers cannot be possible and the ML solution $\hat{\mathbf{d}}$ can be expressed as

$$
\hat{\mathbf{d}}=\underset{\mathbf{d} \in\{0, \mathcal{S}\}}{\operatorname{argmin}}\|\mathbf{y}-\tilde{\mathbf{H}} \tilde{\mathbf{d}}\|^{2}
$$

From (6), it is obvious that a straightforward solution to ML detection of SMXGFDM-IM scheme is extremely complex. Therefore, low complexity solutions are needed.

\subsection{2. $M M S E$ Detection of $S M X-G F D M-I M$}

As a suboptimal but less complex alternative to extremely complex bruteforce ML detection of SMX-GFDM-IM, MMSE-based detection can be considered. Based on the system model in (5), MMSE detection can be performed by

$$
\left[\begin{array}{c}
\mathbf{z}_{1} \\
\mathbf{z}_{2} \\
\vdots \\
\mathbf{z}_{T}
\end{array}\right]=\left(\widetilde{\mathbf{H}}^{\mathrm{H}} \widetilde{\mathbf{H}}+\sigma_{w}^{2} \mathbf{I}\right)^{-1} \widetilde{\mathbf{H}}^{\mathrm{H}} \mathbf{y}
$$

$190 \quad$ where $\mathbf{z}_{t}=\left[\mathbf{z}_{t}^{1}{ }^{\mathrm{T}}, \mathbf{z}_{t}^{2^{\mathrm{T}}}, \ldots, \mathbf{z}_{t}^{L^{\mathrm{T}}}\right]^{\mathrm{T}}$, for $t=1,2, \ldots, T$ and $\mathbf{z}_{t}^{l}$, for $l=1,2, \ldots, L$, is a $u \times 1$ vector. Then, the MMSE solution $\hat{\mathbf{d}}_{t}^{l}$ for $l$ th GFDM-IM subblock of $t$ th transmit antenna is given by

$$
\hat{\mathbf{d}}_{t}^{l}=\underset{\mathbf{d}_{t}^{l} \in\{0, \mathcal{S}\}}{\operatorname{argmin}}\left\|\mathbf{z}_{t}^{l}-\mathbf{d}_{t}^{l}\right\|^{2} .
$$

After this point, detected GFDM-IM subblocks are concatenated to construct the estimated GFDM-IM blocks $\hat{\mathbf{d}}_{t}$ for each transmit antenna and GFDM-IM demappers retrieve the original information bits in each branch of the receiver. 


\subsubsection{Near-ML Detection of SMX-GFDM-IM}

In [15, a combination of SD and SIC has been proposed for SMX-GFDM system utilizing QR decomposition and it has been reported that the proposed method achieves near-ML performance in the high-signal-to-noise (SNR) regimes. In [40, this method has been adapted for GFDM-based space and frequency IM schemes. QR decomposition-based near-ML detection is also applicable for SMX-GFDM-IM system. The MMSE extended QR-decomposition (MMSE-QRD) [4] of $\tilde{\mathbf{H}}$ is given as

$$
\left[\begin{array}{c}
\tilde{\mathbf{H}} \boldsymbol{\Pi} \\
\sigma_{n} \mathbf{I}_{N T}
\end{array}\right]=\mathbf{Q R}=\left[\begin{array}{l}
\mathbf{Q}_{1} \\
\mathbf{Q}_{2}
\end{array}\right] \mathbf{R}
$$

where $\mathbf{Q}$ is an $(N T+N R) \times N T$ unitary matrix which is partitioned into $N R \times$

$N T$ matrix $\mathbf{Q}_{1}$ and $N T \times N T$ matrix $\mathbf{Q}_{2}, \mathbf{R}$ is an $N T \times N T$ upper triangular matrix, $\boldsymbol{\Pi}$ permutes the columns of $\tilde{\mathbf{H}}$ prior to decomposition in order to realize deinterleaving. Then, multiplying the received signals with $\mathbf{Q}_{1}^{\mathrm{H}}$ yields

$$
\tilde{\mathbf{y}}=\mathbf{Q}_{1}^{\mathrm{H}} \mathbf{y}=\mathbf{R} \mathbf{d}-\sigma_{n} \mathbf{Q}_{2}^{\mathrm{H}} \mathbf{d}+\mathbf{Q}_{1}^{\mathrm{H}} \mathbf{w}
$$

Here, first term is the useful signal, second and third terms are the remaining interference that can not be removed by the SIC and channel noise, respectively. Ignoring the interference yields

$$
\tilde{\mathbf{y}}=\mathbf{Q}_{1}^{\mathrm{H}} \mathbf{y}=\mathbf{R d}+\tilde{\mathbf{w}}
$$

where $\tilde{\mathbf{w}}=\mathbf{Q}_{1}{ }^{\mathrm{H}} \mathbf{w}$. At this point, with the help of the upper triangular structure of $\mathbf{R}$, starting from the last GFDM-IM subblock of last transmit antenna, the near-ML solution $\hat{\mathbf{d}}_{t}^{l}$ for $l$ th GFDM-IM subblock of $t$ th transmit antenna is given by

$$
\hat{\mathbf{d}}_{t}^{l}=\underset{\mathbf{d}_{t}^{l} \in\{0, \mathcal{S}\}}{\operatorname{argmin}}\left\|\tilde{\mathbf{y}}_{t}^{l}-\mathbf{R}_{t}^{l} \mathbf{d}_{t}^{l}\right\|^{2}
$$

${ }_{215}$ where $\tilde{\mathbf{y}}_{t}^{l}$ is a $u \times 1$ vector constructed from the last $u$ elements of $\tilde{\mathbf{y}}, \mathbf{R}_{t}^{l}$ is a $u \times u$ upper triangular matrix constructed from the last $u$ rows and columns of $\mathbf{R}$ and $\mathbf{d}_{t}^{l}$ is a $u \times 1$ vector. After that, the interference originated from $\mathbf{d}_{t}^{l}$ is 
obtained as

$$
\hat{\mathbf{y}}_{t}^{l}=\mathbf{R}_{:, u} \hat{\mathbf{d}}_{t}^{l},
$$

where the subscripts $u$ and : denote the last $u$ and all elements (column/rows)

of the subscripted object, respectively, and canceled:

$$
\tilde{\mathbf{y}} \leftarrow \tilde{\mathbf{y}}-\hat{\mathbf{y}}_{t}^{l} .
$$

Then, the system size is reduced by $u$ which is shown as

$$
\tilde{\mathbf{y}} \leftarrow \tilde{\mathbf{y}}_{\bar{u}}, \quad \mathbf{R} \leftarrow \mathbf{R}_{\bar{u}, \bar{u}},
$$

where, the subscript $\bar{u}$ denote the all but the last $u$ elements of the subscripted object, and detection operation continues to the next GFDM-IM subblock until all subblocks are detected. After this point, original information bits are 225 retrieved as explained in Section 2.2 .2

\section{Complexity Analysis}

Computational complexities of SMX-GFDM-IM receivers with MMSE, MMSEQRD and ML detectors are analyzed in terms of number of complex multiplications $(\mathrm{CMs})$ performed in total and presented in Tables 1, 2 and 3, respectively. Here, $\Phi_{J \times I}$ and $\Psi_{J \times I}$ are used for $J \times I$ matrices, $\phi_{J \times 1}$ and $\psi_{J \times 1}$ stand for $J \times 1$ vectors, $(\cdot)^{+}$and $Q R(\cdot)$ perform pseudo-inversion and $\mathrm{QR}$ decomposition, respectively. The complexity orders in terms of total number of CMs are presented in Table 4. From Table 4, it is observed that SMX-GFDM-IM receiver with ML detector has the highest computational complexity and the computational complexity of MMSE and MMSE-QRD detection-based receivers are approximately the same.

\section{Simulation Results and Discussion}

In this section, uncoded and coded BER performances of the SMX-GFDMIM schemes are evaluated by Monte Carlo simulations for Rayleigh multipath 
Table 1: Computational Complexity of MMSE Detection-Based SMX-GFDM-IM Receiver

\begin{tabular}{|l||c|c|c|}
\hline Process & Operation & Execution Count & Complexity (CMs) \\
\hline \hline Forming $\widetilde{\mathbf{H}}$ & $\Phi_{N \times N} \Psi_{N \times N^{*}}$ & $R T$ & $N_{\mathrm{Ch}} N^{2} R T$ \\
\hline MMSE detect. & $\left(\Phi_{N R \times N T}{ }^{\mathrm{H}} \Phi_{N R \times N T}+\Psi_{N T \times N T}\right)^{-1} \Phi_{N R \times N T}{ }^{\mathrm{H}} \phi_{N R \times 1}$ & 1 & $2 N^{3} T^{2} R+N^{3} T^{3}+N^{2} R T$ \\
\hline Block deintlv. & $\mathrm{No}_{\mathrm{CM}}$ & $\mathrm{R}$ \\
\hline SMX-IM demod. & $\left.\min \left(\| \phi_{u \times 1}-\psi_{u \times 1}\right) \|^{2}\right)$ & $(N / u) c Q^{v} T$ & 0 \\
\hline
\end{tabular}

${ }^{*}$ In every row of $\mathbf{H}_{r, t}$, only $N_{C h}$ out of $N$ elements are non-zero.

Table 2: Computational Complexity of MMSE-QRD Detection-Based SMX-GFDM-IM Receiver

\begin{tabular}{|l||c|c|c|}
\hline Process & Operation & Execution Count & Complexity (CMs) \\
\hline \hline Forming $\widetilde{\mathbf{H}}$ & $\Phi_{N \times N} \Psi_{N \times N}^{\dagger}$ & $R T$ & $N_{\mathrm{Ch}} N^{2} R T$ \\
\hline MMSE-QRD & $Q R\left(\Phi_{(N R+N T) \times N T}\right)^{\dagger \dagger}$ & 1 & $2 N^{3} T^{2}(T+R)$ \\
\hline Modified recv. vector & $\Phi_{N R \times N T^{\mathrm{H}} \phi_{N R \times 1}}$ & 1 & $N^{2} R T$ \\
\hline ML detect. & $\min \left(\left\|\phi_{u \times 1}-\left(\Phi_{u \times u} \psi_{u \times 1}\right)\right\|^{2}\right)^{\dagger \dagger \dagger}$ & $(N / u) c Q^{v} T$ & $(N / u)\left(c Q^{v} T(u v+u)\right)$ \\
\hline SIC & $\phi_{N T \times 1}-\Phi_{N T \times u} \psi_{u \times 1}^{\dagger \dagger \dagger}$ & $(N / u) T$ & $N^{2} T^{2} v / u$ \\
\hline
\end{tabular}

${ }^{\dagger}$ In every row of $\mathbf{H}_{r, t}$, only $N_{C h}$ out of $N$ elements are non-zero.

${ }^{\dagger}$ The modified Gram-Schmidt orthogonalization algorithm requires $2 m n^{2}$ complex multiplication to compute the QR-factorization of an $m \times n$ matrix.

${ }^{\dagger \dagger \dagger}$ In $\psi$, only $v$ complex elements are nonzero.

Table 3: Computational Complexity of ML Detection-Based SMX-GFDM-IM Receiver

\begin{tabular}{|l||c|c|c|}
\hline Process & Operation & Execution Count & Complexity (CMs) \\
\hline \hline Forming $\widetilde{\mathbf{H}}$ & $\Phi_{N \times N} \Psi_{N \times N^{\ddagger}}$ & $R T$ & $N_{\mathrm{Ch}} N^{2} R T$ \\
\hline ML Detection. & $\min \left(\left\|\phi_{N T \times 1}-\left(\Phi_{N R \times N T} \psi_{N T \times 1}\right)\right\|^{2}\right)^{\ddagger \ddagger}$ & $\left(c Q^{v}\right)^{(T N / u)}$ & $\left(c Q^{v}\right)^{(T N / u)}\left(\left(N^{2} T R v / u\right)+N T\right)$ \\
\hline
\end{tabular}

$\ddagger$ In every row of $\mathbf{H}_{r, t}$, only $N_{C h}$ out of $N$ elements are non-zero.

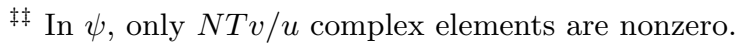


Table 4: Summary of The Computational Complexity of Three Different Receiver Structures

\begin{tabular}{|l||c|}
\hline Receiver & Complexity $(C M s)$ \\
\hline \hline MMSE & $\sim \mathcal{O}\left(N^{3}\left(T^{3}+2 T^{2} R\right)\right)$ \\
\hline MMSE-QRD & $\sim \mathcal{O}\left(N^{3}\left(2 T^{3}+2 T^{2} R\right)\right)$ \\
\hline ML & $\left(c Q^{v}\right)^{(T N / u)}\left(\left(N^{2} T R v / u\right)+N T\right)$ \\
\hline
\end{tabular}

Table 5: System Parameters

\begin{tabular}{|l||c|c|}
\hline Description & Parameter & Value \\
\hline \hline Number of subcarriers & $K$ & 128 \\
\hline Number of subsymbols & $M$ & 5 \\
\hline Pulse shaping filter & $g$ & $\mathrm{RC}$ \\
\hline Roll-off factor & $a$ & 0.5 \\
\hline Length of cyclic prefix & $N_{\mathrm{CP}}$ & 32 \\
\hline Number of channel taps for EPA channel & $N_{\mathrm{Ch}}$ & 7 \\
\hline
\end{tabular}

fading channels with Extended Pedestrian A (EPA) channel model [45. Raised cosine $(\mathrm{RC})$ with a roll-off factor $(a)$ of 0.5 is chosen as a pulse shape for the GFDM prototype filter. System parameters are shown in Table 5.

Figs. 4 and 5 compare the uncoded BER performances of the SMX-GFDMIM schemes with SMX-OFDM and SMX-GFDM schemes for $2 \times 2$ and $4 \times 4$ 245 MIMO configurations, respectively, using BPSK transmission. In order to select the active subcarrier indices, the look-up table in Table 6 is used. From Fig. 4 for a $2 \times 2$ MIMO configuration and at a BER value of $10^{-4}$, it is observed that the SMX-GFDM-IM scheme with MMSE-QRD detection achieves $4.5 \mathrm{~dB}$ better BER performance than SMX-OFDM and SMX-GFDM schemes with MMSEQRD detection. The performance improvement of SMX-GFDM-IM scheme with MMSE-QRD detection with respect to SMX schemes arises from the coding gain due to enhanced distance spectrum of IM. On the other hand, for SMX- 
Table 6: A look-up table example for $u=4, v=2$ and $p_{i}=2$

\begin{tabular}{|c||c||c|c|}
\hline Bits & Indices & \multicolumn{3}{|c|}{ subblocks } \\
\hline \hline$\left[\begin{array}{ll}0 & 0\end{array}\right]$ & $\{1,2\}$ & {$\left[\begin{array}{llll}s_{\chi} & s_{\zeta} & 0 & 0\end{array}\right]^{T}$} \\
\hline$\left[\begin{array}{ll}0 & 1\end{array}\right]$ & $\{2,3\}$ & {$\left[\begin{array}{llll}0 & s_{\chi} & s_{\zeta} & 0\end{array}\right]^{T}$} \\
\hline$\left[\begin{array}{ll}1 & 0\end{array}\right]$ & $\{3,4\}$ & {$\left[\begin{array}{llll}0 & 0 & s_{\chi} & s_{\zeta}\end{array}\right]^{T}$} \\
\hline$\left[\begin{array}{ll}1 & 1\end{array}\right]$ & $\{1,4\}$ & {$\left[\begin{array}{llll}s_{\chi} & 0 & 0 & s_{\zeta}\end{array}\right]^{T}$} \\
\hline
\end{tabular}

GFDM-IM scheme, MMSE-QRD detection method provides $3.5 \mathrm{~dB}$ better BER performance than MMSE detection. The performance improvement of MMSEQRD detection method with respect to MMSE method is the result of the joint MIMO detection, GFDM and IM demodulation of the active subcarrier indices and the data symbols in a near-optimal manner. In Fig. 5 for a $4 \times 4$ MIMO configuration, while BER performance gain of the SMX-GFDM-IM scheme with MMSE-QRD detection with respect to SMX-OFDM and SMX-GFDM schemes with MMSE-QRD detection is approximately the same with BPSK transmission, BER performance gain of the SMX-GFDM-IM scheme with MMSE-QRD detection with respect to SMX-GFDM-IM scheme with MMSE detection is increased to $6 \mathrm{~dB}$. The reason behind this improvement is the increased diversity order of the MMSE-QRD detector with the number of receive antennas. Table

7 shows the number of CMs required to implement the configurations given in Figs. 4 and 5. From Table 7 it is observed that SMX-GFDM-IM receiver with ML detection has infeasible computational complexity as expected and the BER performance improvement of the MMSE-QRD detector with respect to MMSE detector is provided with negligible increase in computational complexity.

In GFDM, a single CP is used for entire block consisting $K$ subcarriers with $M$ timeslots on each of it. Therefore, GFDM provides a higher spectral efficiency with respect OFDM due to reduced overhead of CP. For the configurations in Figs. 4 and 5 GFDM schemes provide 19\% spectral efficiency with respect to OFDM schemes. Here, the spectral efficiency of SMX-IM schemes is 


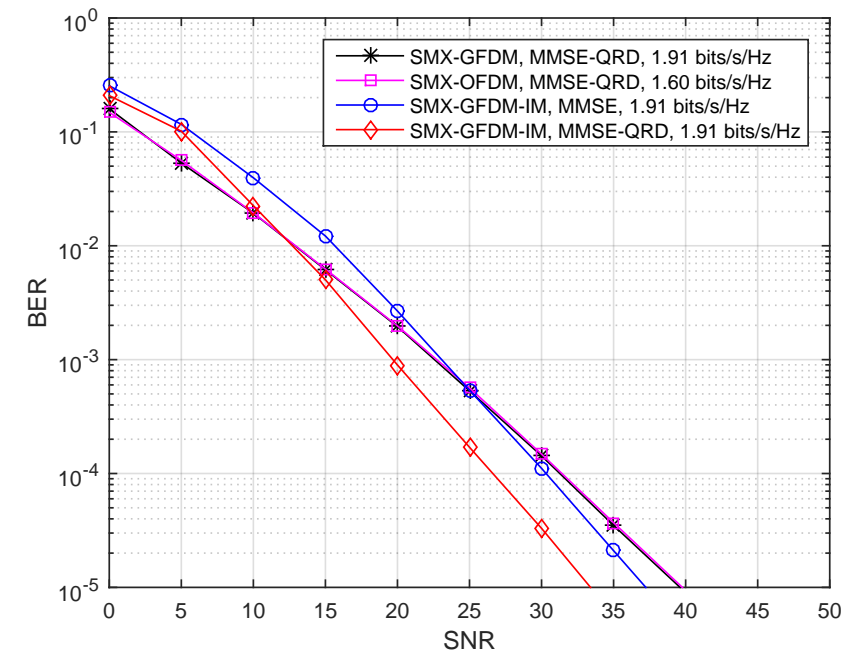

Figure 4: Uncoded BER performance of MMSE and MMSE-QRD detection methods for $2 \times 2$ SMX and SMX-IM schemes using BPSK transmission.

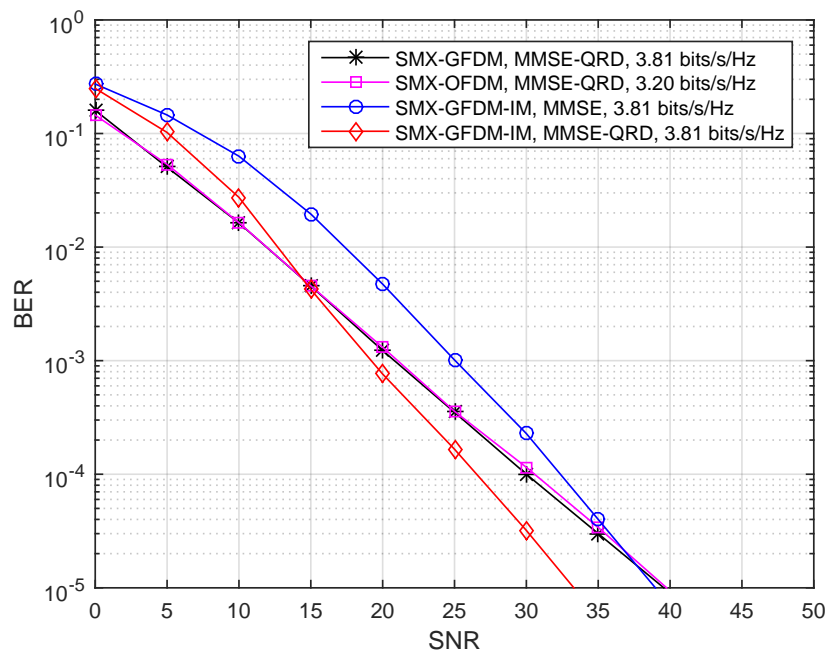

Figure 5: Uncoded BER performance of MMSE and MMSE-QRD detection methods for $4 \times 4$ SMX and SMX-IM schemes using BPSK transmission.

given by $p /\left(N+N_{\mathrm{CP}}\right)$, and the spectral efficiency of SMX schemes is given by $N \log _{2}(Q) /\left(N+N_{\mathrm{CP}}\right)$. 


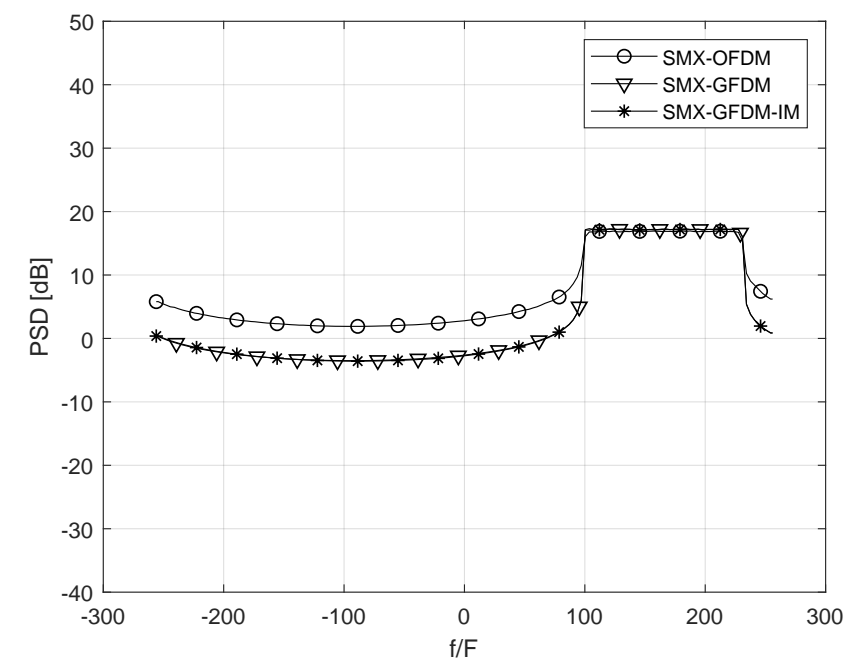

Figure 6: OOB emission comparison of SMX and SMX-IM schemes using RC pulse shaping filter with a roll-off factor 0.5 for BPSK transmission.

Table 7: The Total Number of CMs for SMX-GFDM-IM Receivers using BPSK

\begin{tabular}{|l||c|c|c|}
\hline Ant. Config. & $M M S E$ & $M M S E-Q R D$ & $M L$ \\
\hline \hline $2 \times 2$ & $6.31 \times 10^{9}$ & $8.40 \times 10^{9}$ & $3.64 \times 10^{487}$ \\
\hline $4 \times 4$ & $5.04 \times 10^{10}$ & $6.72 \times 10^{10}$ & $2.95 \times 10^{1162}$ \\
\hline
\end{tabular}

Fig. 6 shows the OOB emission comparison of SMX and SMX-IM schemes using RC pulse shaping filter with a roll-off factor of 0.5 for BPSK transmission. From Fig. 6, it is observed that GFDM schemes provides $6 \mathrm{~dB}$ OOB emission suppression with respect to OFDM scheme due to pulse shaping of each subcarrier with RC filter. Note that, there is not any difference on OOB emission of SMX-GFDM and SMX-GFDM-IM schemes as expected.

Figs. 7 and 8 show the uncoded BER performance of the SMX-GFDM-IM schemes along with the SMX-OFDM and SMX-GFDM schemes for $2 \times 2$ and $4 \times 4$ MIMO configurations, respectively, using 4-QAM transmission. Here, the active subcarrier indices are determined using the look-up table in Table 
Table 8: A look-up table example for $u=4, v=3$ and $p_{i}=2$

\begin{tabular}{|c||c||ll|}
\hline Bits & Indices & \multicolumn{3}{c|}{ subblocks } \\
\hline \hline$\left[\begin{array}{ll}0 & 0\end{array}\right]$ & $\{1,2,3\}$ & {$\left[\begin{array}{llll}s_{\chi} & s_{\zeta} & s_{\delta} & 0\end{array}\right]^{T}$} \\
\hline$\left[\begin{array}{ll}0 & 1\end{array}\right]$ & $\{1,2,4\}$ & {$\left[\begin{array}{llll}s_{\chi} & s_{\zeta} & 0 & s_{\delta}\end{array}\right]^{T}$} \\
\hline$\left[\begin{array}{ll}1 & 0\end{array}\right]$ & $\{1,3,4\}$ & {$\left[\begin{array}{llll}s_{\chi} & 0 & s_{\zeta} & s_{\delta}\end{array}\right]^{T}$} \\
\hline$\left[\begin{array}{ll}1 & 1\end{array}\right]$ & $\{2,3,4\}$ & {$\left[\begin{array}{llll}0 & s_{\chi} & s_{\zeta} & s_{\delta}\end{array}\right]^{T}$} \\
\hline
\end{tabular}

Table 9: The Total Number of CMs for SMX-GFDM-IM Receivers using 4-QAM

\begin{tabular}{|l||c|c|c|}
\hline Ant. Config. & MMSE & MMSE-QRD & $M L$ \\
\hline \hline $2 \times 2$ & $6.31 \times 10^{9}$ & $8.40 \times 10^{9}$ & $1.14 \times 10^{873}$ \\
\hline $4 \times 4$ & $5.04 \times 10^{10}$ & $6.72 \times 10^{10}$ & $1.92 \times 10^{1933}$ \\
\hline
\end{tabular}

8. From Figs. 7 and 8 , it is observed that the BER performance gain of the SMX-GFDM-IM schemes with MMSE-QRD detection with respect to other schemes are decreased compared to the gains in BPSK transmission. The reason behind this decrease is the decrease of the $p_{i} / p_{q}$ ratio from 1 to 0.33 . On the other hand, the number of CMs required to implement the configurations given in Figs. 7 and 8 are given in Table 9. From Table 9, it is observed that ML detector has the highest computational complexity as in the BPSK case and the computational complexity of MMSE-QRD detector is higher than the computational complexity of MMSE detector with a negligible level.

Fig. 9 compares the coded BER performance of the SMX-GFDM-IM scheme with SMX-OFDM and SMX-GFDM schemes for $4 \times 4$ MIMO configuration using 16-QAM transmission. For the channel code, the rate $1 / 3$ convolutional code with generator sequence $q=[133,171,165]$ is chosen [46]. In order to select 300 the active subcarrier indices, the look-up table in Table 8 is used. From Fig. 9. at a BER value of $10^{-4}$, it is observed that the SMX-GFDM-IM scheme achieves $2.5 \mathrm{~dB}$ better BER performance than SMX-OFDM and SMX-GFDM 


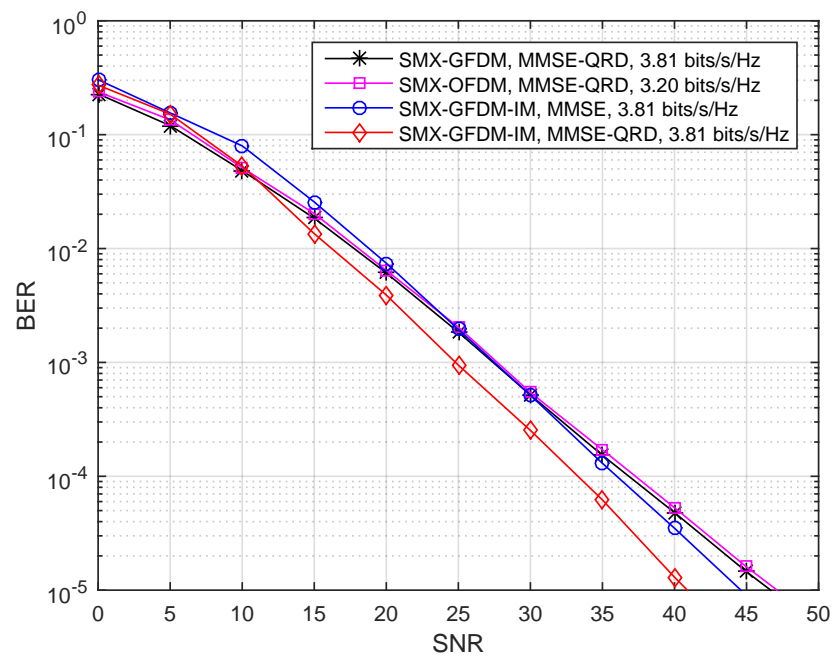

Figure 7: Uncoded BER performance of MMSE and MMSE-QRD detection methods for $2 \times 2$ SMX and SMX-IM schemes using 4-QAM transmission.

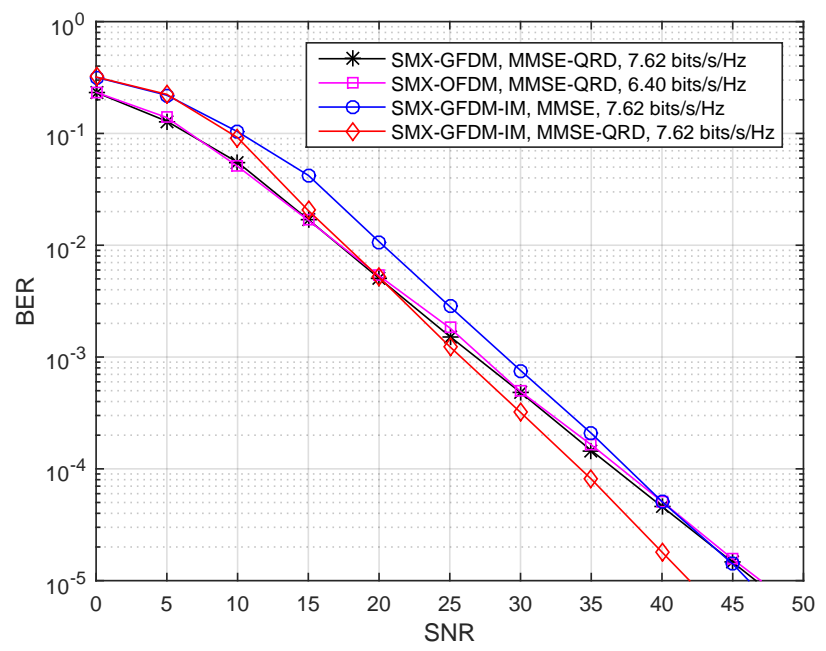

Figure 8: Uncoded BER performance of MMSE and MMSE-QRD detection methods for $4 \times 4$ SMX and SMX-IM schemes using 4-QAM transmission.

schemes. Here, since the $p_{i} / p_{q}$ ratio is decreased to 0.16 , BER performance improvement of the IM scheme is decreased with respect to BPSK and 4-QAM

schemes. Therefore, it can be stated that the BER improvements of the IM 


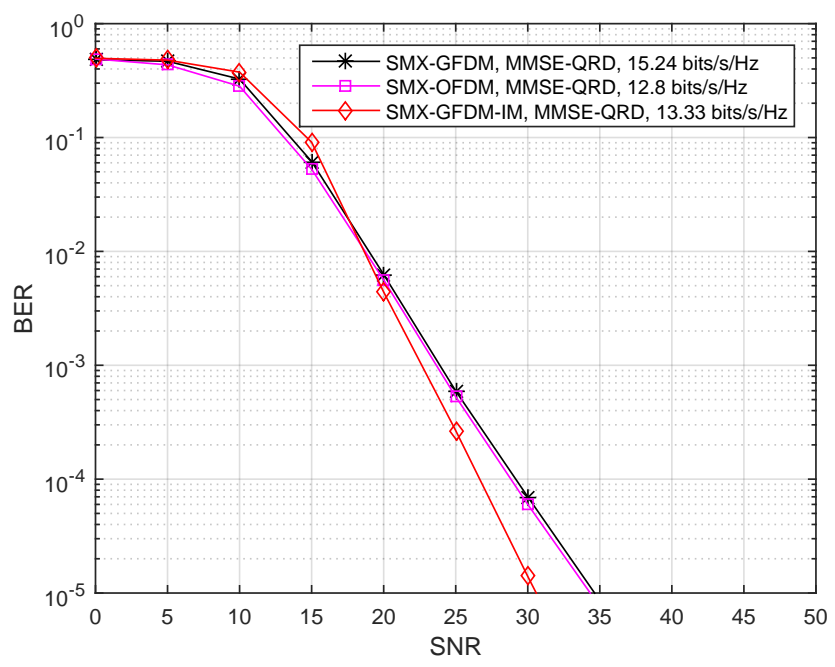

Figure 9: Coded BER performance of $4 \times 4$ SMX and SMX-IM schemes using 16-QAM transmission.

scheme is directly proportional with the amount of information bits conveyed by the indices of the transmit entities as expected. Besides, considering Figs. 5. 8 and 9, it is observed that channel coding improves the BER performance of the SMX-GFDM-IM scheme. Note that, for the system configuration given in Fig. 9, while spectral efficiency of SMX-GFDM-IM scheme is slightly lower than SMX-GFDM scheme due to unused subcarriers, it has still higher spectral efficiency with respect to SMX-OFDM scheme due to reduced overhead of CP.

\section{Conclusion}

In this paper, SMX and IM techniques have been combined with GFDM in order to cope with the challenging technical requirements of beyond $5 \mathrm{G}$ wireless networks. At the transmitter side, each transmit antenna transmits its own GFDM-IM block, and at the receiver side, GFDM-IM blocks are detected and demodulated using a novel MMSE-QRD-based near-optimum detector. We have investigated the error performance, OOB emission, spectral efficiency and computational complexity of the proposed scheme via computer simulations. It 
has been demonstrated that the proposed SMX-GFDM-IM scheme achieves better error performance with respect to classical SMX-OFDM and SMX-GFDM schemes and has a lower OOB emission and better spectral efficiency advantages with respect to SMX-OFDM scheme because of digitally pulse shaping of each subcarrier and reduced overhead of cyclic prefix. Based on these advantages, SMX-GFDM-IM scheme can be considered as a viable PHY scheme for beyond $5 \mathrm{G}$ wireless networks.

\section{References}

[1] J. G. Andrews et. al., What will 5G be?, IEEE J. Sel. Areas Commun. 32 (6) (2014) 1065-1082.

[2] Study on scenarios and requirements for next generation access technologies, tech. rep. 38.913 V15.0.0, 3GPP, 2018.

[3] G. Wunder et. al., 5GNOW: Non-orthogonal, asynchronous waveforms for future mobile applications, IEEE Commun. Mag. 52 (2) (2014) 97-105.

[4] P. Banelli et. al., Modulation formats and waveforms for 5G networks: Who will be the heir ofOFDM?: An overview of alternative modulation schemes for improved spectral efficiency, IEEE Signal Process. Mag. 31 (6) (2014) 80-93.

[5] NR, physical layer, general description, tech. spec. 38.201 V15.0.0, 3GPP 2017.

[6] Study on new radio (NR) access technology, tech. spec. 38.912 V15.0.0, 3GPP, 2018.

[7] N. Michailow, M. Matthe, I. Gaspar, A. Caldevilla, L. Mendes, A. Festag, G. Fettweis, Generalized frequency division multiplexing for 5th generation cellular networks, IEEE Trans. Commun. 62 (9) (2014) 3045-3061.

[8] M. Matthe, L. L. Mendes, G. Fettweis, Space-time coding for generalized frequency division multiplexing, 20th Eur. Wireless Conf. (2014) 1-5. 
[9] M. Matthe, L. L. Mendes, I. Gaspar, N. Michailov, D. Zhang, G. Fettweis, Widely linear estimation for space-time-coded GFDM in low latency applications, IEEE Trans. Commun. 63 (11) (2015) 4501-4509.

[10] M. Matthe, L. L. Mendes, I. Gaspar, N. Michailov, D. Zhang, G. Fettweis, Multi-user time-reversal STC-GFDMA for future wireless networks, EURASIP J. Wireless Commun. Netw. 2015 (1) (2015) 1-8.

[11] D. Zhang, L. L. Mendes, M. Matthe, N. Michailov, G. Fettweis, Expectation propagation for near-optimum detection of MIMO-GFDM signals, IEEE Trans. Wireless Commun. 15 (2) (2016) 1045-1062.

[12] D. Zhang, L. L. Mendes, M. Matthe, G. Fettweis, A markov chain Monte Carlo algorithm for near-optimum detection of MIMO-GFDM signals, IEEE Personal, Indoor and Mobile Radio Commun., (PIMRC'15) (2015) $1-5$.

[13] J. Zhong, G. Chen, J. Mao, S. Dang, P. Xiao, Iterative frequency domain equalization for MIMO-GFDM systems, IEEE Access 6 (2018) 1938619395.

[14] N. Tunali, M. Wu, C. Dick, C. Studer, S. Jose, Linear large-scale MIMO data detection for 5G multi-carrier waveform candidates, Asilomar Conf. on Signals, Systems, and Computers (2015) 1-5.

[15] M. Matthe, I. Gaspar, D. Zhang, G. Fettweis, Near-ML detection for MIMO-GFDM, Proc. 82nd IEEE Veh. Technol. Conf. Fall (2015) 1-2.

[16] M. Matthe, D. Zhang, G. Fettweis, Sphere-decoding aided SIC for MIMOGFDM: Coded performance analysis, Int. Symp. on Wireless Commun. Systems (ISWCS) (2016) 165-169.

[17] M. Matthe, D. Zhang, G. Fettweis, Iterative detection using MMSE-PIC demapping for MIMO-GFDM systems, IEEE European Wireless (EW'16) (2016) 1-7. 
[24] D. Tsonev, S. Sinanovic, H. Haas, Enhanced subcarrier index modulation (SIM) OFDM, IEEE GLOBECOM Workshops (2011) 728-732.

[25] T. Mao, Z. Wang, Q. Wang, S. Chen, L. Hanzo, Dual-mode index modulation aided OFDM, IEEE Access 5 (2017) 50-60.

[26] M. Wen, E. Basar, Q. Li, B. Zheng, M. Zhang, Multiple-mode orthogonal frequency division multiplexing with index modulation, IEEE Trans. Commun. 65 (9) (2017) 3892-3906.

[27] E. Basar, OFDM with index modulation using coordinate interleaving, IEEE Wireless Commun. Lett. 4 (4) (2015) 381 - 384. 
[28] R. Fan, Y. J. Yu, Y. L. Guan, Generalization of orthogonal frequency division multiplexing with index modulation, IEEE Trans. Wireless Commun. 14 (10) (2015) $5350-5359$.

[29] M. Wen, Y. Zhang, J. Li, E. Basar, F. Chen, Equiprobable subcarrier activation method for OFDM with index modulation, IEEE Commun. Lett. 20 (12) (2016) $2386-2389$.

[30] M. Wen, B. Ye, E. Basar, Q. Li, F. Ji, Enhanced orthogonal frequency division multiplexing with index modulation, IEEE Trans. Wireless Commun. 16 (7) (2017) $4786-4801$.

[31] H. Zhang, L. L. Yang, L. Hanzo, Compressed sensing improves the per410 formance of subcarrier index-modulation-assisted OFDM, IEEE Access 4 (2016) $7859-7873$.

[32] E. Basar, Multiple-input multiple-output OFDM with index modulation, IEEE Signal Process. Lett. 22 (12) (2015) 2259 - 2263.

[33] T. Datta, H. S. Eshwaraiah, A. Chockalingam, Generalized space-and-

[35] B. Zheng, M. Wen, E. Basar, F. Chen, Multiple-input multiple-output OFDM with index modulation: Low-complexity detector design, IEEE Trans. Signal Process. 65 (11) (2017) 2758 - 2772.

[36] E. Basar, Index modulation techniques for $5 \mathrm{G}$ wireless networks, IEEE Commun. Mag. 54 (7) (2016) 168-175.

${ }_{425}$ [37] E. Ozturk, E. Basar, H. Cirpan, Spatial modulation GFDM: A low complexity MIMO-GFDM system for 5G wireless networks, Proc. 4th IEEE Int. Black Sea Conf. Commun. Networking (2016) 1-5. 
[38] E. Ozturk, E. Basar, H. Cirpan, Generalized frequency division multiplexing with index modulation, Proc. IEEE GLOBECOM Workshops (2016) $1-6$.

[39] E. Ozturk, E. Basar, H. Cirpan, Generalized frequency division multiplexing with space and frequency index modulations, Proc. 5th IEEE Int. Black Sea Conf. Commun. Networking (2017) 1-6.

[40] E. Ozturk, E. Basar, H. Cirpan, Generalized frequency division multiplexing with flexible index modulation, IEEE Access 5 (2017) 24727 - 24746.

[41] E. Ozturk, E. Basar, H. Cirpan, Generalized frequency division multiplexing with flexible index modulation numerology, IEEE Signal Process. Lett. 25 (10) (2018) 1480-1484.

[42] Y. K. Chang, F. B. Ueng, A novel turbo GFDM-IM receiver for MIMO communications, International Journal of Electronics and Communications 87 (2018) 22-32.

[43] E. Yuzgecoglu, M. Jorswieck, Transceiver design for GFDM with index modulation in multi-user networks, Proc. WSA 2018, 22nd International ITG Workshop on Smart Antennas (2018) 43-48.

${ }_{445}$ [44] D. Wubben, R. Bohnke, V. Kuhn, K. Kammeyer, MMSE extension of VBLAST based on sorted QR decomposition, 2003 IEEE 58th Vehicular Technology Conference (2003) 508-512.

[45] Base station (BS) radio transmission and reception, tech. spec. 36.104 V14.4.0, 3GPP, 2017.

450 [46] Multiplexing and channel coding, tech. spec. 36.212 V14.3.0, 3GPP, 2017. 